\title{
Design on Network Management System of Community Movements Based on ASP and VB.NET Technology
}

\author{
Yuanhai Wang \\ School Physical Education, Yuxi Normal University, Yuxi, 653100, China \\ yuanhaiwang2014@yeah.net
}

Keywords: ASP.NET; Evaluation system; Multilevel encryption; Simulation test

\begin{abstract}
Strengthen the protection of personal information, learning prevention method of network information attack. Through scientific means to protect the privacy and self interest of Network Management System of Community Movements system is the professional knowledge. Based on the digital certificate we do encryption on encryption of the digital signature, according to the Mark off model we c establish transfer matrix of data integrity, so as to realize the encryption of students' evaluation information system. Multidimensional network information security platform needs to improve confidentiality failure, dynamic integrity and distributed availability of data security in addition to the client's safety and the master-slave structure safety.
\end{abstract}

\section{Introduction}

At early time the web server sends the basic static page, accessing to web with HTML format. The disadvantage is that after completion of the static page editor, it will cause the content of the page not having dynamic changes [1,2]. If you need to change the page again, it will cost a lot of time. In order to solve this problem, dynamic page emerges as the times require, each dynamic pages have an existing database[3]. But with the further development of information technology, the mainstream information system begins to use the B/S three-layer structure mode, using ASP technology to achieve the basic operation of web. But it can only be used on the Windows platform [4,5]. This development model reduces the workload of the client computer, and keeps cost as low as possible. More and more users select this development mode.

ASP combines the components of HTML, Script and ActiveX together, which can form a application running on the server. And according to the requirements of the customers, it sends special HTML page to the client browser [6,7]. The basic request of the data is completed by the ODBC or OLEDB link. Its core component is the ADO technology [8]. Through the ADO command, it completes increasing, deleting, changing, checking the whole dynamic database.

\section{Optimization of Students' Evaluation of Information System}

Network Management System of Community Movements information system based on ASP.NET mainly uses ASP.NET, ADO.NET and SQL Server2008 [9]. The front end of students' evaluation information system develops the Web by ASP.NET. The backstage has SQL Server2008 as database support. Its structure is as shown below.

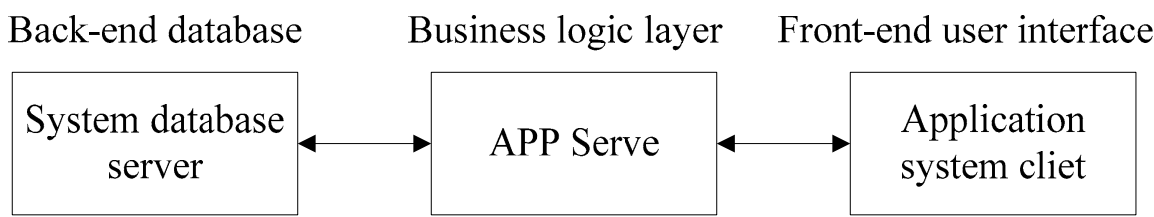

Fig. 1 Three-layer structure of Network Management System of Community Movements information system

As shown in Figure 1, the core layer-three layer structure is business logic relationship, which provides interactive operation for the users. And at the same time it will send dynamic data of background to the front end users. Each module of three-layer structure is connected to each other 
[10]. Base on three-layer system structure, we use ASP.NET and ADO.NET to develop students' evaluation information system.

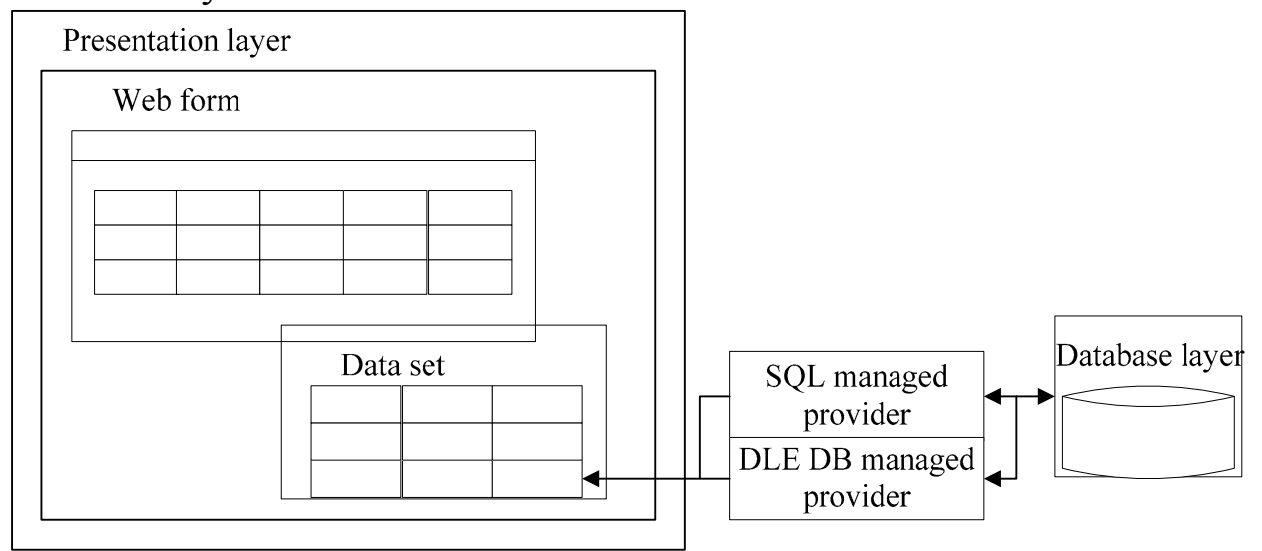

Fig. 2 Integration framework of ASP.NET, ADO.NET

As Figure 2 shows, managed provider is a bridge between application and data sources of ADO.NET. Role permission management of evaluation system is very important, before entering the system it must first authenticate [11]. According to the different user permissions, it provides different services. The processing flow of encryption information is as shown below.

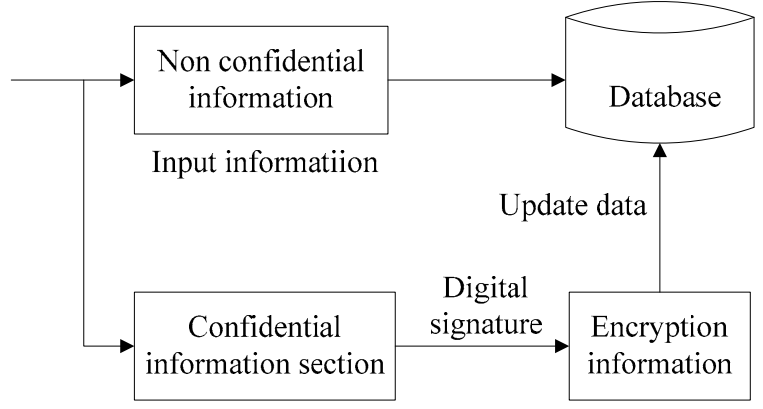

Fig. 3 The processing flow of encryption information

As shown in Figure 3, information entered the database consists of two parts, confidential and non confidential information [12]. The encryption information is through digital signature, the basic parameters in signature schemes are $(M, S, K, S I G, V E R)$, Where: $M$-news set; $S$-signature set; $K$ keys set, including private and public keys; $S I G$-signature algorithm set; $V E R$-signature verification algorithm set. The signature verification algorithm is:

verk: $\mathrm{M} * \mathrm{~S} \rightarrow\{$ True, False $\}$.

verk $(x \neg y)=\left\{\begin{array}{l}\text { True, } y=\operatorname{sigk}(x) \\ \text { False, } y \neq \operatorname{sigk}(x) \text {. }\end{array}\right.$

In the formula, after the signature verifier receives $(m, s)$, he will calculate $\operatorname{Verk}(x, y)$, if $\operatorname{Verk}(x, y)=$ True, the signature is true, or it is wrong. The optimization of digital signature is as shown below.

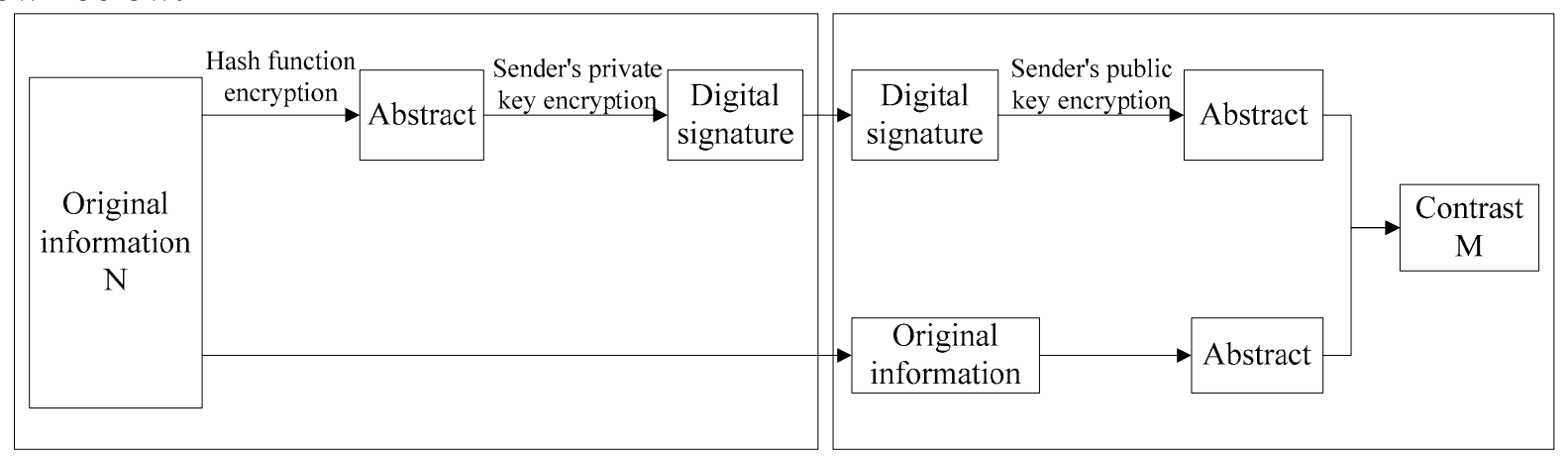

Fig.4 The optimization of digital signature

As shown in Figure $4, N$ is the state value of data node, and the collection of state is $\mathrm{p}=\left\{\mathrm{p}_{1}, p_{2}, \ldots, p_{n}\right\}$. At time $\mathrm{t}$, state of data node is $z_{i}, z_{i} \in \mathrm{p}, z_{i}$ is state value of data nod from 
observer. And the state observation value set is $E=\left\{e_{1}, e_{2}, \cdots e_{m}\right\}$. At time $t$, state observation value of data node from observer is $o_{t}, o_{t \in E}$. In the actual monitoring data, the emergency process is as shown below.

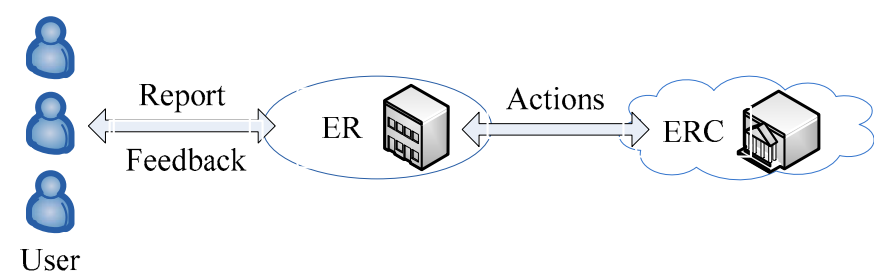

Fig. 5 Emergency model of students' evaluation system

As shown in Figure 5, according to the Mark off model, we can establish transfer matrix of data integrity state, it can be expressed as below:

$$
A=\left[\begin{array}{cccc}
a_{\mathrm{p}_{1} \cdot p_{1}} & a_{p_{1} \cdot p_{2}} & \cdots & a_{p_{1}, p_{n}} \\
a_{p_{2} \cdot p_{1}} & a_{p_{2} \cdot p_{2}} & \cdots & a_{p_{2} \cdot p_{n}} \\
\ldots & \cdots & \cdots & \cdots \\
a_{p_{n} \cdot p_{1}} & a_{p_{n} \cdot s_{2}} & \cdots & a_{p_{n} \cdot p_{n}}
\end{array}\right] .
$$

$a_{p 1 . p t}$ is the probability from the current state $p_{i}$ to state $p_{j}$. The independence of the data integrity is:

$$
p\left(o_{1}, o_{1}, \ldots, o_{1} \mid Z_{1}, Z_{1}, \ldots, Z_{1}, \theta\right)=\prod_{t-1}^{T} p\left(o_{t} \mid Z_{t}, \theta\right) .
$$

As shown in formula (4), through the node verification of digital certificate, any state of data integrity monitoring probability $a_{\mathrm{p}_{0} \cdot \mathrm{p}_{t}}$ from state $\mathrm{p}_{0}$ to state $\mathrm{p}_{i}$. Any state of data integrity is the probability $a_{s_{i}, s_{0}}$ from state $s_{i}$ to state $s_{0}$. By controlling the probability of the test data, it ensures the integrity of teachers' confidential information.

\section{Simulation Test}

In order to verify the performance of encryption, we use computer to do simulation at the same time [13]. In order to make the whole verification scientific, we use several test software as shown in the following table.

Table 1. Relevant test software

\begin{tabular}{|c|c|c|}
\hline Detection tool & $\begin{array}{c}\text { Detection tool, port scanning tool } \\
\text { tools }\end{array}$ & Vulnerability scanning tools \\
\hline Tcpdump (Linux); Ethereal & PortScan (DOS); Nmap 4.03 & Nessus 3.0.5 (Linux); \\
$\begin{array}{c}\text { 0.10.11 (Windows); Sniffer } \\
\text { Pro 4.7.5(Windows); Snort }\end{array}$ & (Liunx); SuperScan 4.0 & X-Scan v3.3 (Windows); \\
2.8.0.1(Windows); & (Windows); & \\
\hline
\end{tabular}

Table 2. Platform and services

\begin{tabular}{|c|c|c|c|}
\hline & Intel S3000PT HPC Server & Intel UPStorage Server & Application Server \\
\hline Network & $\begin{array}{c}\text { Intel® Dual-Ports 1000M } \\
\text { Netadapter }\end{array}$ & $\begin{array}{c}\text { Intel } \AA \text { Dual-Ports 1000M } \\
\text { Netadapter }\end{array}$ & $\begin{array}{c}\text { Intel } \AA \text { Dual-Ports } \\
1000 \mathrm{M} \text { Netadapter }\end{array}$ \\
\hline Platform & $\begin{array}{c}\text { Windows Server 2003,Suse } \\
10\end{array}$ & $\begin{array}{c}\text { Windows Server 2003,Suse } \\
10\end{array}$ & $\begin{array}{c}\text { Windows Server } \\
2003, \text { Suse 10 }\end{array}$ \\
\hline Accessories & Server Management & Server Management & Related Test Software \\
\hline
\end{tabular}




\begin{tabular}{|c|c|c|c|}
\hline Services & Email, File and Website & Database, FTP & Application \\
\hline
\end{tabular}

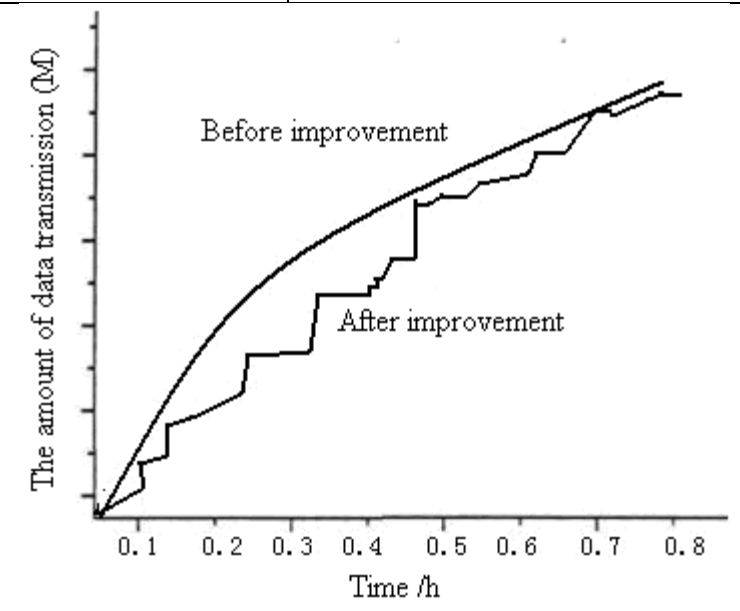

Fig. 6 The results of simulation

As shown in Figure 6, from the data integrity simulation based on digital certificate, in the time of simulation, it identifies the intrusion extreme point of data node accurately. According to the peak value, we can find threat coefficient of evaluation system [14]. At the same time we can dig threat factors of information integrity. It provides the basis to establish preventive measures for evaluation system.

\section{Summary}

The development of information technology change rapidly, which breaks the traditional method of browsing information. Open distribution interface has brought certain risks to the information security. Solving security problems of information system is the hot topic in this field. In this paper we first analyze mainstream B/S system architecture of the current information system, and analyze the principle of ASP.NET visiting Web database. On this basis we do multilevel encryption processing on Network Management System of Community Movements information system, so as to realize the optimization of information security. In order to verify the security performance, we use software to do simulation testing. The results show that the method has strong practicability, good encryption effect. It solves encryption of Network Management System of Community Movements information system to a certain extent.

\section{References}

[1] He Dequan, Tang Zhengjun, Li Jianhua. The intrusion detection technology [M]. Tsinghua University press, 2012: 50-62.

[2] Kun Wang, Lihua Zhou, Zhen Cai, Zengxin Li, A Disaster Recovery System Model in an e-Government System [J]. Sixth International Conference on Parallel and Distributed Computing Applications and Technologies, 2010(7): 247-250.

[3] Wang Xiao, Xue Zhi. Wang Yijun. Game analysis of intrusion detection system based on honeypot [J]. Information and communication security, 2011(12): 55-61.

[4] $\mathrm{K} \mathrm{Yu,} \mathrm{Hu} \mathrm{Liang,} \mathrm{Zhang} \mathrm{Xiaohui.} \mathrm{A} \mathrm{distributed} \mathrm{intrusion} \mathrm{detection} \mathrm{system} \mathrm{model} \mathrm{based} \mathrm{on} \mathrm{agent}$ and honeypot [J]. Journal of Jilin University (SCIENCE EDITION), 2011(3): 34-41.

[5] Joan C.Steyaert. Measuring the performance of electronic government services [J]. Information \& Management, 2011(41):369-375.

[6] Qin Xueli. Web application technology design [M]. Tsinghua University press, 2012: 50-65. 
[7] Wang Jian. Design and implementation of teaching evaluation system based on Web [J]. Journal of Chengde Teachers College for nationalities, 2012(2): 78-84.

[8] Zhou Qiang, Yang Geng. Data fusion algorithm of protecting data integrity and privacy in WSNs [J]. Computer Application Research, 2013(2): 2-10.

[9] Zhang Lei, Zhou Jifeng, Zhang Qiang. Research on system software reliability demonstration test [J]. Computer and digital engineering, 2010(6): 23-29.

[10]Li Yan. Study on information security technology based on digital certificate [J]. The application of information technology, 2012(7): 45-51.

[11] Maocai Wang. An efficient generation method of elliptic curve for pairing-based cryptosystems. Proc. of 2010 International Conference on Machine Vision and Human-machine Interface, MVHI2010, Kaifeng, China.

[12] Bob Gourley. Cloud Computing and Net Centric Operations [J]. Department of Defense Information Management and Information Technology Strategic Plan, 2011(18): 1023-1030.

[13] Hu Guangjun. Research on the strategies of acquiring information based on dynamic game [J]. Technology review, 2012(4): 34-41.

[14] Gaffney, J.E, Jr. Ulvila, J.W. Evaluation of intrusion detectors: a decision theory approach [J]. Security and Privacy, 2011. S\&P 2011, 2011(6): 50-61. 